\title{
Actividad física, ejercicio y nutrición en niños y adolescentes
}

\author{
Physical activity, exercise and nutrition in children and adolescents \\ Atividade física, exercício e nutrição em crianças e adolescentes
}

Pablo Rosselli ${ }^{*}$, Harold Arévalo ${ }^{2}$

Recibido: 30 de mayo de 2019. Aceptado para publicación: 5 de agosto de 2019. https://doi.org/10.35454/rncm.v2n2.007

\section{Resumen}

Una actividad física adecuada y una nutrición balanceada son fundamentales para el buen desarrollo físico y mental de niños y adolescentes. Infortunadamente, el estilo de vida actual induce al sedentarismo y a los malos hábitos alimentarios. Esto se traduce en sobrepeso y aumento del riesgo de adquirir enfermedades crónicas no transmisibles que pueden acortar la expectativa de vida. Aunque existen políticas de salud pública eficientes para disminuir la carga en los sistemas de salud, aún queda mucho por hacer para contrarrestar esta epidemia de sedentarismo y de mala nutrición que producen millones de muertes al año.

La Organización Mundial de la Salud (OMS) da unas recomendaciones sobre la intensidad, duración, frecuencia y tipo de ejercicio que deben hacer los niños de acuerdo con su edad con el fin de lograr una adecuada salud mental, ósea, muscular y metabólica.

Palabras clave: actividad física, ejercicio, sobrepeso, nutrición balanceada, enfermedades crónicas no transmisibles

\section{Summary}

A balanced nutrition and intense physical activities are necessary for the normal development of children and adolescents. Unfortunately, current lifestyle generates sedentary life and unhealthy nutrition. These two produce overweight and an increased risk of acquiring chronic noncommunicable diseases which in the long term create illness and shorten lifespan. Although certain public policies are efficient to reduce the burden of sedentary habits and malnutrition, there is still much to do in order to reduce the epidemics of unhealthy life habits responsible of millions of deaths each year.

World health organization gives recommendations for the necessary physical activity needed in different ages in order to obtain an adequate health.

Keywords: physical activity, exercise, overweight, balanced nutrition, chronic non-communicable diseases.

\section{Resumo}

A atividade física adequada e a nutrição equilibrado são essenciais para o bom desenvolvimento físico e mental de crianças e adolescentes. Infelizmente, o estilo de vida atual induz o sedentarismo e a maus hábitos alimentares. Isso se traduz em excesso de peso e aumento do risco de adquirir doenças crônicas não transmissíveis que podem reduzir a expectativa de vida. Embora existam políticas de saúde pública eficientes para diminuir o ônus sobre os sistemas de saúde, ainda há muito a ser feito para combater essa epidemia de sedentarismo e má nutrição que produzem milhões de mortes por ano.

A Organização Mundial de Saúde (OMS) dá recomendações sobre a intensidade1, duração, frequência e tipo de exercício que as crianças devem fazer de acordo com a sua idade, a fim de alcançar uma saúde mental óssea, muscular e metabólica adequadas.

Palavras-chave: atividade física, exercício, excesso de peso, nutrição balanceada, doenças crônicas não transmissíveis.
1. Fundación Cardioinfantil, Bogotá, D.C., Colombia.

2. Medicina del Deporte, Universidad El Bosque, Bogotá, D. C., Colombia.
Correspondencia: *Pablo Rosselli dr.rosselli@gmail.com 


\section{INTRODUCCIÓN}

Los efectos benéficos del ejercicio físico y una nutrición adecuada en la salud de niños y adolescentes son innegables. El balance entre estos dos garantiza una homeostasis de los diferentes sistemas corporales encargados del crecimiento, neuro desarrollo y maduración de la personalidad. Infortunadamente, el mundo moderno es sedentario y se alimenta mal. Esto ha dado origen a una serie de enfermedades en donde se incluyen el sobrepeso y la obesidad que aumentan el costo de la salud y acorta la expectativa de vida.

Si los especialistas encargados de la nutrición de niños y adolescentes, y los demás profesionales del área de la salud competentes no toman medidas en el asunto, nuestro país seguirá el rumbo de otros que han perdido la batalla contra el sedentarismo y la mala nutrición.

\section{ANÁLISIS Y DISCUSIÓN}

El $40 \%$ del peso corporal de hombres y mujeres está constituido por músculo. Esta conformación no es producto del azar, sino el resultado de un proceso evolutivo de miles de años de una especie diseñada para moverse. El movimiento trae consigo efectos benéficos sobre prácticamente todos los sistemas corporales que se resumen en la tabla 1. Sin embargo, hay un dato sobrecogedor y preocupante: hace 100 años el ser humano consumía $80 \%$ de las calorías ingeridas en ejercicio físico, hoy solo gasta $5 \%^{(1)}$. Este cambio en el balance energético que altera la composición corporal es producto de varios factores.

Para empezar, los vertiginosos avances tecnológicos de las últimas décadas han hecho que hombres y mujeres se entretengan y se comuniquen desde la perversa comodidad de su hogar. La televisión, los teléfonos móviles y demás dispositivos electrónicos invitan a la inmovilidad y a la reclusión en espacios reducidos. En algunos casos, el contacto con la tecnología empieza desde edades tan tempranas como el año de edad; el uso de estos aparatos por muchas horas al día crea adicción y trastornos del comportamiento y, lo más grave, impiden que el niño se ejercite. A su vez, puede generar alteración del sueño, otro elemento fundamental para el normal desarrollo del niño y el adolescente.

$\mathrm{Si}$ a esto se le suma el hecho de que en la mayor parte de la gente vive en ciudades contaminadas, inseguras y con problemas de movilidad el panorama se hace más desalentador. Nunca antes en la historia de la humanidad el hombre había sido tan sedentario, y las dietas tan abundantes, pobres en micronutrientes y vitaminas y a la vez ricas en grasas saturadas. La combinación mortal de sedentarismo y malnutrición da origen a los desórdenes que se conocen como enfermedades crónicas no transmisibles (hipertensión arterial, diabetes tipo 2, cáncer) en donde se incluyen además el sobrepeso y la obesidad. Se calcula que en 2010 habían 42 millones de niños con sobrepeso en el mundo, de los cuales 35 millones vivían en países en vías de desarrollo ${ }^{(2)}$. El problema es de tal dimensión, que la expectativa de vida de hombres y mujeres en muchos países del mundo se ha reducido.

Para que las intervenciones en salud pública sean eficientes y se logre mejorar la actividad física de los niños se debe trabajar en varios frentes: la atención primaria en salud, los medios de comunicación, la comunidad, el colegio y las políticas medioambientales (figura 1). Un ejemplo de esta última es la ciclovía en la ciudad de Bogotá los días festivos en la que se promueve el ejercicio de manera divertida, sociable, diversa y motivante, y ha demostrado ser sostenible a través de los años ${ }^{(3,4)}$.

Tabla 1. Efectos benéficos del ejercicio. Tomado de Rosselli Cock $P^{(3)}$.

\begin{tabular}{|c|c|}
\hline Fisiológicos & Psicológicos, académicos y sociales \\
\hline Mejora el movimiento coordinado y la velocidad de reacción. & Disminuye el estrés, la ansiedad y la depresión. \\
\hline Favorece el crecimiento de los huesos y músculos y mejora la & Ayuda a superar la timidez y mejora la autoestima. \\
\hline densidad mineral ósea. & Frena impulsos excesivos y mejora la calidad del sueño. \\
\hline Mejora los niveles de glucosa y colesterol en sangre. & Enriquece el lenguaje verbal y escrito, así como el desempeño \\
\hline Disminuye la adiposidad central y total. & escolar. \\
\hline Incrementa la capacidad aeróbica. & Crea hábitos y disciplina. \\
\hline Mejora la postura. & Fomenta el trabajo grupal por encima del individualismo. \\
\hline Fortalece el sistema inmune. & $\begin{array}{l}\text { Les hará reconocer, aceptar y respetar que existe alguien que } \\
\text { sabe más que ellos. }\end{array}$ \\
\hline
\end{tabular}




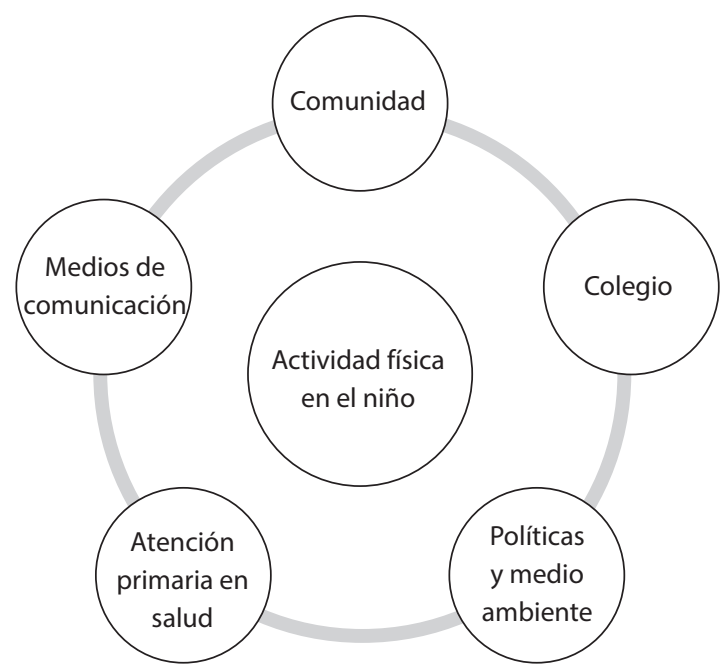

Figura 1. Elementos en la promoción de la actividad física en niños y adolescentes. Tomado de Rosselli Cock $\mathrm{P}^{(3)}$.

El pensum escolar de educación física y ejercicio de la mayoría de instituciones educativas, no solo en Colombia sino en otros países del mundo, es insuficiente. Con la falsa convicción de que se debe hacer énfasis en el aprendizaje de las materias tradicionales, relegan a un nivel secundario la actividad física el ejercicio y el deporte. Es este un enfoque desacertado. Muchas publicaciones coinciden en resaltar la importancia del movimiento en el aprendizaje, la cognición y el desarrollo de la memoria.

La dieta del niño que se ejercita ha de suplir la energía y los nutrientes suficientes para reponer las pérdidas de glucógeno hepático y muscular, garantizar el crecimiento, mantener una adecuada composición corporal y proveer los macronutrientes y micronutrientes necesarios ${ }^{(3)}$.

Y no solo hay que pensar en el niño sano. También deben incluirse los niños con condiciones especiales. En la medida en que mejoran las condiciones de vida de los niños sanos, también mejoran las de los niños con ciertas circunstancias especiales. Con el paso de los años mejora la sobrevida de niños, con síndrome de Down, parálisis cerebral y otras situaciones de discapacidad que también merecen atención en cuanto a las recomendaciones nutricionales y de ejercicio ${ }^{(3)}$.

\section{RECOMENDACIONES GENERALES DE ACTIVIDAD FÍSICA Y NUTRICIÓN}

En el ámbito mundial existen diferentes recomendaciones de tiempo estimado para realizar actividad física en los niños que supera el de la población adulta. Este tiempo, que en promedio es de entre 90 a 120 minutos diarios de actividad física estructurada (con seguimiento, orden y desarrollo), suele incumplirse.

La Organización Mundial de la Salud emitió recientemente unas nuevas recomendaciones para los menores de 5 años. En los mayores se siguen las mismas recomendaciones formuladas por esa misma entidad en 2010:

\section{Los lactantes (menores de un año)}

- Deben estar físicamente activos varias veces al día de diferentes formas, al menos 30 minutos en posición prona, repartidos a lo largo del día mientras estén despiertos $^{(5)}$.

- No deben permanecer sujetos durante más de una hora seguida (carritos, coche, sillas), no se recomienda que pasen tiempo frente a pantallas. En momentos de inactividad se recomienda que un cuidador les lea o cuente cuentos ${ }^{(5)}$.

- Deben tener de 14 a 17 horas (0 a 3 meses de edad) o de 12 a 16 horas (4 a 11 meses de edad) de sueño de buena calidad ${ }^{(5)}$.

\section{Los niños de 1 a 2 años}

- Deben pasar por lo menos 180 minutos realizando diversos tipos de actividad física de cualquier intensidad a lo largo del día ${ }^{(5)}$.

- No deben permanecer sujetos durante más de una hora seguida, ni permanecer sentados durante periodos largos de tiempo. No se recomienda que niños de 1 año pasen tiempo en actividades sedentarias ante una pantalla. Para los niños de 2 años, el tiempo dedicado a actividades sedentarias frente a una pantalla no debe exceder 60 minutos; cuanto menos mejor ${ }^{(5)}$.

- Deben tener de 11 a 14 horas de sueño de buena calidad $^{(5)}$.

\section{Los niños de 3 a 4 años}

- Deben pasar por lo menos 180 minutos realizando diversos tipos de actividad física de cualquier intensidad a lo largo del día. Incluido al menos 60 minutos de actividades físicas de intensidad moderada a elevada ${ }^{(5)}$.

- No deben permanecer sujetos durante más de una hora seguida, ni permanecer sentados durante periodos largos de tiempo. El tiempo dedicado a actividades sedentarias frente a una pantalla no debe exceder 60 minutos; cuanto menos mejor ${ }^{(5)}$. 
- Deben tener de 10 a 13 horas de sueño de buena calidad $^{(5)}$.

\section{Los niños de 5 a 17 años}

Deben hacer actividad física vigorosa y actividades de alta intensidad entre una y tres horas al día. Este es el tiempo apropiado para un adecuado gasto energético, para una buena maduración metabólica $(6-8 \mathrm{Kcal} /$ $\mathrm{Kg} / \mathrm{día})$. Aquí se deben incorporar actividades que promuevan el fortalecimiento muscular y óseo por lo menos tres veces por semana. Se debe trabajar en flexibilidad, coordinación, velocidad de reacción y resaltar que hacia los 15 años se presenta un descenso en el tiempo que los adolescentes dedican a actividades físicas de entre moderada y vigorosa intensidad ${ }^{(3)}$.

La actividad física en niños y adolescentes deberá estar acorde con sus procesos fisiológicos de madurez y adaptación. Un error que se comete en algunos lugares de formación en deporte, es la mezcla de diferentes edades y capacidades funcionales bajo una misma intensidad o exigencia deportiva. Por esta razón, el deporte de alto rendimiento infantil no es lo ideal, mensaje que debería cambiar en padres, entrenadores y formadores. El ejercicio y el deporte en este grupo etario debe verse como una herramienta en el desarrollo psicosocial y neurológico ${ }^{(1,3)}$.

La composición corporal del niño se debe tener en cuenta en la práctica de la actividad física y el ejercicio. El peso corporal es el resultado del peso de todos los tejidos corporales, que se dividen en dos grandes grupos: masa libre de grasa (FFM, por su sigla en inglés) y masa grasa (FM, por su sigla en inglés). Estos tejidos cambian en las etapas del ciclo de la vida; el tejido FFM crece en forma acelerada en la época del estirón puberal, mientras que la FM cambia de manera progresiva. La velocidad de estos cambios está relacionada con las respuestas hormonales de cada individuo. Por lo tanto, el rendimiento físico, desempeño muscular, fuerza, potencia y su metabolismo energético, serán producto del momento del desarrollo infantil ${ }^{(3)}$.

Es una prioridad que los niños y adolescentes tengan aportes nutricionales de buena calidad que cumplan con los requerimientos basales que garanticen un desarrollo ideal. No es correcto exigir el consumo de alimentos extraordinarios o costosos cuando es imposible acceder a ellos, por lo que se debe ser práctico y eficaz en su prescripción. Los niños requieren el consumo de todos los grupos de alimentos con algunas excepciones en condiciones especiales de salud. Debe hacerse vigi- lancia especial a las familias o personas practicantes de conductas nutricionales alternativas como el vegetarianismo, entre otras, pues existe el riesgo de deficiencia de algunos nutrientes y oligoelementos, no se debe seguir la "moda de la nutrición" del momento. Están proscritas las bebidas energizantes por sus demostrados efectos negativos en la salud, entre ellos la de producir arritmias cardíacas. La mejor forma de hidratación en niños y adolescentes es a través del agua ${ }^{(3)}$.

Aunque no es el tema de esta revisión, se debe resaltar la evaluación pre participativa en los niños por la posibilidad de muerte súbita, circunstancia que se ha hecho frecuente con la práctica masiva del ejercicio y el deporte. Esta evaluación debería tenerla el grueso de los participantes en deporte y ejecutada por personal idóneo; existen diferentes cuestionarios pre participativos tanto del Colegio Americano de Medicina del Deporte como de la Academia Americana de Pediatría. Sin embargo, la mayor información proviene de una buena historia clínica (interrogatorio personal y familiar) y un buen examen físico. Si teniendo en cuenta esto, el experto considerará ampliar la información, para lo cual se podrá apoyar en exámenes complementarios. La incidencia de esta entidad depende del número de participantes en deporte por el número de horas de práctica deportiva ${ }^{(3)}$.

\section{CONCLUSIÓN}

Nutricionistas, médicos y todas las personas encargadas de la salud de niños y adolescentes deben saber los beneficios de la práctica regular del ejercicio, y están en la obligación de trabajar en equipo. Si bien estos conocimientos hacen parte de una rama especializada de la medicina (medicina del deporte), su prescripción como un signo vital más es obligatorio como medida preventiva de las enfermedades crónicas no transmisibles.

\section{Financiación}

El presente artículo no tuvo financiación.

\section{Conflicto de intereses}

Los autores declaran no tener conflicto de intereses.

\section{Declaración de autoría}

Los autores participaron por igual en la concepción y redacción del artículo. De igual forma declaran que revisaron de artículo y validaron su versión final. 


\section{Referencias bibliográficas}

1. Duperly J, Lobelo F. Prescripción del ejercicio. Una guía para recomendar actividad física a cada paciente. Primera edición. Bogotá, Colombia: Ediciones de la U; 2015.

2. Blair SN. Physical activity: the biggest public health problem of the 21st century. Br J Sports Med. 2009;43(1):1-2.

3. Rosselli Cock P. La Actividad Física, el Ejercicio y el Deporte en Niños y Adolescentes. Recomendaciones en la salud y en la enfermedad. Primera edición. Bogotá, Colombia. Editorial Médica Panamericana; 2018.
4. Instituto Distrital de Recreación y Deporte (IDRC). Programa Muévete Bogotá [Internet]. Bogotá, Colombia.. [Fecha de consulta: 24 de mayo de 2019]. Disponible en: https://guiatramitesyservicios.bogota.gov.co/tramite-servicio/programamuevete-bogota/

5. World Health Organization. Guidelines on physical activity, sedentary behavior and sleep for children under 5 years of age [Internet]. Geneva: World Health Organization, 2019. [Fecha de consulta: 24 de mayo 2019] Disponible en: http://www. who.int/iris/handle/10665/311664. 